\title{
The dynamic regulation of nitrogen and phosphorus in the early phase of fermentation improves the erythromycin production by recombinant Saccharopolyspora erythraea strain
}

\author{
Qiang Zhang ${ }^{1}$, Yong Chen ${ }^{1,2}$, Ming Hong ${ }^{1}$, Yang Gao ${ }^{3}$, Ju Chu*, Ying-ping Zhuang ${ }^{1}$ and Si-liang Zhang ${ }^{1}$
}

\begin{abstract}
Background: Erythromycin production often has concern with the consumption rate of amino nitrogen and phosphate, especially in the early fermentation phase. The dynamic regulation of nitrogen and phosphorus was put forward based on the comprehensive analysis of the contents of phosphorus and nitrogen in different nitrogen sources as well as the relations between nitrogen consumption and phosphorus consumption.
\end{abstract}

Results: Firstly, the unstable nitrogen source, corn steep liquor, was substituted with the stable nitrogen source, yeast powder, with little effects on erythromycin production. Secondly, feeding phosphate in the early fermentation stage accelerated the consumption of amino nitrogen and ultimately increased erythromycin production by approximately 24\% as compared with the control (without feeding potassium dihydrogen phosphate). Thirdly, feeding phosphate strategy successfully applied to $500 \mathrm{~L}$ fermenter with the final erythromycin concentration of $11839 \mathrm{U} / \mathrm{mL}$, which was $17.3 \%$ higher than that of the control. Finally, the application of condensed soy protein (a cheap nitrogen source with low phosphorus content) combined with phosphate feed strategy led to a 13.0\% increase of the erythromycin production as compared with the control (condensed soy protein, without feeding potassium dihydrogen phosphate).

Conclusions: Appropriately feeding phosphate combined with rational nitrogen regulation in the early fermentation phase was an effective way to improve erythromycin production.

Keywords: Erythromycin; Potassium dihydrogen phosphate; Feeding strategy; Yeast powder; Condensed soy protein

\section{Background}

Erythromycin is a kind of polyketide antibiotic produced by Saccharopolyspora erythraea through industrial fermentation. Recently, erythromycin has received much attention because its semi-synthetically modified derivatives, such as azithromycin, roxithromycin, and clarithromycin, are widely used in the treatment of infectious diseases [1,2]. The high requirement of erythromycin market stimulates the research on the improvement of the production and productivity of erythromycin. It has been reported that the genetic engineering technology,

\footnotetext{
* Correspondence: juchu@ecust.edu.cn

'State Key Laboratory of Bioreactor Engineering, East China University of Science and Technology, 130 Meilong Road, P.O. box 329, Shanghai 200237, People's Republic of China

Full list of author information is available at the end of the article
}

such as Saccharopolyspora erythraea mutB knockout and artificial attB site, have been applied to improve erythromycin production $[3,4]$. The variation of the medium composition and fermentation conditions also successfully improved the erythromycin productivity $[5,6]$. Zou et al. reported that feeding corn steep liquor could regulate oxygen uptake rate (OUR) at a suitable level in the early phase of fermentation, elevate the intracellular levels of lactic acid, pyruvic acid, citric acid, and propionic acid, and ultimately enhance the metabolic flux of erythromycin biosynthesis [7]. It is well known that the quality of corn steep liquor is unstable due to its production process and the difficulty to be stored [8]. This led to the severe fluctuation of erythromycin production on industrial scale. In this study, yeast powder, a stable nitrogen source, was 
used to replace corn steep liquor, so that the erythromycin production could be maintained at a stable level. Our data showed that the erythromycin production was little affected by the substitution of corn steep liquor with yeast powder. Furthermore, it was found that phosphorus content limited the consumption rate of amino nitrogen.

Compared with organic phosphorus, the concentration of inorganic phosphorus can be controlled quantitatively. In most cases, phosphorus is used as a crucial growth-limiting nutrient in antibiotic fermentations. The concentration of soluble phosphate generally fell in the range of 0.0003 to $0.3 \mathrm{~mol} / \mathrm{L}$. When the concentration of soluble phosphate was higher than $0.01 \mathrm{~mol} / \mathrm{L}$, the biosynthesis of many antibiotics would be suppressed [9-11]. It was reported that the initial phosphate concentration of 0.0061 to $0.0096 \mathrm{~mol} / \mathrm{L}$ was beneficial for the cell growth in the aminoglycoside antibiotic JI-20A fermentation [12] and feeding phosphorus could provide optimal conditions for penicillin $\mathrm{G}$ biosynthesis [13]. In our previous work, it was found that erythromycin A production was increased by $8.7 \%$ through the rational regulations of phosphate salt, soybean meal, and ammonium sulfate [14]. However, few studies, to the best of our knowledge, focused on the effects of initial phosphorus and feeding phosphorus in the early stage of fermentation on the erythromycin fermentation. Hence, the effects of adding and feeding potassium dihydrogen phosphate in the early fermentation phase on the erythromycin fermentation were investigated.

\section{Methods}

\section{Microorganism and culture conditions}

The recombinant erythromycin-producing strains Saccharopolyspora erythraea ZL1004 is preserved in our laboratory [15].

Preliminary seed medium contains $(\mathrm{g} / \mathrm{L})$ the following: starch 30 , dextrin 40 , soybean meal $20, \mathrm{CaCO}_{3} 5, \mathrm{NaCl}$ 3 , and $\left(\mathrm{NH}_{4}\right)_{2} \mathrm{SO}_{4} 2$.

Secondary seed medium contains $(\mathrm{g} / \mathrm{L})$ the following: starch 30, dextrin 30, soybean meal 30, corn steep liquor $10, \mathrm{CaCO}_{3} 5$, and antifoam agent $1.25 \mathrm{~mL}$.

In the fermentation in 50-L fermenter, preliminary seed culture was transferred into a $15-\mathrm{L}$ fermenter containing $8 \mathrm{~L}$ of the secondary seed medium and cultivated at $34^{\circ} \mathrm{C}$ for $41 \mathrm{~h}$. Then, the secondary seed culture was transferred into a 50-L fermenter containing $30 \mathrm{~L}$ of fermentation medium and cultivated at $34^{\circ} \mathrm{C}$ for $190 \mathrm{~h}$. The fermenter was an agitated bioreactor equipped with three turbine impellers and devices capable of monitoring more than 14 online measurable parameters (FUS-50, Shanghai Guoqiang Bioengineering Equipment Co., Ltd., Shanghai, China) $[9,16]$. DO (dissolved oxygen) was monitored with polarographic DO electrode (Mettler-Toledo, Greifensee,
Switzerland) and controlled in 40-60\% of air saturation by adjusting agitation and aeration during fermentation.

\section{Experimental methods}

The substitution of corn steep liquor with yeast powder

The original fermentation medium $(\mathrm{g} / \mathrm{L})$ consisted of starch 35, dextrin 5, soybean meal 30, corn steep liquor 18 , ammonium sulfate $3.0, \mathrm{NaCl} 2.0, \mathrm{CaCO}_{3} 7.0$, and antifoam agent $1.9 \mathrm{~mL}$. For the experiment group, corn steep liquor was replaced by $10 \mathrm{~g} / \mathrm{L}$ yeast powder. The nitrogen content of $18 \mathrm{~g} / \mathrm{L}$ corn steep liquor $(0.734 \mathrm{~g} / \mathrm{L})$ was equal to that of $10 \mathrm{~g} / \mathrm{L}$ yeast powder $(0.736 \mathrm{~g} / \mathrm{L})$ based on their total nitrogen amount detected by the Kjeldahl method [17].

\section{Adding and feeding potassium dihydrogen phosphate in the early stage of fermentation.}

Strategy 1: adding potassium dihydrogen phosphate to the initial medium. Potassium dihydrogen phosphate was added before the sterilization of medium. The concentrations of potassium dihydrogen phosphate in the medium were set at 0.08 and $0.12 \mathrm{~g} / \mathrm{L}$, respectively, which were determined based on the difference of soluble phosphate concentration between yeast powder medium $(0.07 \mathrm{~g} / \mathrm{L})$ and corn steep liquor medium $(0.14 \mathrm{~g} / \mathrm{L})$.

Strategy 2: Feeding potassium dihydrogen phosphate in the early stage of fermentation The levels of soluble phosphate and amino nitrogen were chosen as the indicator for switching on and switching off phosphate feeding. When soluble phosphate concentration was about $0.04 \mathrm{~g} / \mathrm{L}$, phosphate feed began. When amino nitrogen concentration was near $0.18 \mathrm{~g} / \mathrm{L}$, phosphate feed was terminated. The solution of potassium dihydrogen phosphate $(10 \mathrm{~g} / \mathrm{L})$ was fed at the rate of $10 \mathrm{~g} / \mathrm{h}$ in mode 3 and $20 \mathrm{~g} / \mathrm{h}$ in mode 4 , respectively.

The scale-up of phosphate feed on 500-L fermenter scale Preliminary seed medium and secondary seed medium were the same to the media used for $50 \mathrm{~L}$ fermentation. The fermentation medium was the yeast powder medium. Preliminary seed culture was transferred into a $100-\mathrm{L}$ fermenter containing $60 \mathrm{~L}$ of the secondary seed medium and cultivated at $34^{\circ} \mathrm{C}$ for $41 \mathrm{~h}$. Then the secondary seed culture was transferred into a $500-\mathrm{L}$ fermenter containing $300 \mathrm{~L}$ of fermentation medium and cultivated at $34^{\circ} \mathrm{C}$ for $190 \mathrm{~h}$ (Shanghai Guoqiang Bioengineering Equipment Co., Ltd., Shanghai, China). The solution of potassium dihydrogen phosphate $(10 \mathrm{~g} / \mathrm{L})$ was fed at a rate of $100 \mathrm{~g} / \mathrm{h}$ when soluble phosphate concentration fell to about $0.055 \mathrm{~g} / \mathrm{L}$ and ceased to feed when amino nitrogen concentration was close to $0.18 \mathrm{~g} / \mathrm{L}$. 


\section{The fermentation with condensed soy protein as nitrogen source}

The condensed soy protein medium $(\mathrm{g} / \mathrm{L})$ consisted of starch 35, dextrin 5, soybean meal 30, condensed soy protein 7 , ammonium sulfate $3.0, \mathrm{NaCl} 2.0, \mathrm{CaCO}_{3} 7.0$, and antifoam agent $1.9 \mathrm{~mL}$. The phosphate feed strategy was the same to that of mode 4.

\section{Analytical methods}

Fermentation broth was centrifuged at 4,000 rpm for 10 min and PMV (packed mycelium volume) was the percentage of the precipitation $(v / v)$. The concentration of amino nitrogen was detected by formol titration method [7]. Total sugar concentration was assayed by Fehling method after acid hydrolysis [18]. Soluble phosphate level and total phosphorus content were measured with molybdenum blue method [19].

The erythromycin titer was measured by the modified colorimetric method [20]. The fermentation supernatant was collected after centrifugation and poured into the equal volume of $\mathrm{K}_{2} \mathrm{CO}_{3}$ solution (0.35\%). Then, this solution was extracted with the equal volume of butyl acetate. Extracted erythromycin (oil phase) was mixed with the $0.1 \mathrm{~mol} / \mathrm{L}$ hydrochloric acid. The aqueous phase fraction was separated with great care and further mixed with sulfate acid $(18 \mathrm{~mol} / \mathrm{L})$ for $3 \mathrm{~min}$. Then, the absorbance of this mixed solution was measured at $498 \mathrm{~nm}$ with a spectrophotometer. The standard curve of erythromycin titer was obtained using erythromycin A standard sample ( $97 \%$ purity). The erythromycin titer of fermentation broth was calculated according to the standard curve $\left(y=241.97 x+15.71, R^{2}=0.998\right)$.

\section{Results and discussion}

The erythromycin fermentation with yeast powder as the easily metabolized nitrogen source

Owing to the instability of corn steep liquor, the substitution of corn steep liquor with yeast powder was investigated. The results showed that the erythromycin production of yeast powder medium was reached to $9,746 \mathrm{U} / \mathrm{mL}$ at $192 \mathrm{~h}$, which was similar to that with corn steep liquor medium
$(9,444 \mathrm{U} / \mathrm{mL})$. This result indicated that yeast powder was suitable to replace corn steep liquor for the erythromycin production.

By comparing the process parameters between the medium with yeast powder and the medium with corn steep liquor, the significant differences were observed that the fermentation with yeast powder medium had a much lower concentration of soluble phosphate and a much higher amino nitrogen level during the early fermentation stage as compared with that using corn steep liquor medium (Figure 1A, B). These phenomena could result from the difference in terms of the ingredients between yeast powder and corn steep liquor. The total nitrogen contents in the two media were the same because the substitution of corn steep liquor with yeast powder was based on the equal nitrogen principle. It was well known that the soluble phosphate played an important role in fermentation process optimization and erythromycin biosynthesis. The data shown that the phosphorus content in yeast powder medium $(0.07 \mathrm{~g} / \mathrm{L})$ was only half of that in corn steep liquor medium $(0.14 \mathrm{~g} / \mathrm{L})$. Hence, it was worthwhile to investigate the effects of phosphorus on the fermentation and the erythromycin biosynthesis. In addition, the organic acids in corn steep liquor and the microelements in yeast powder should possibly play a role in the cell growth. However, they may have a looser correlation to the levels of phosphate in the early fermentation stage as compared with phosphorus. Hence, the effects of adding and feeding potassium dihydrogen phosphate on the fermentation were investigated.

\section{The effects of the addition of potassium dihydrogen phosphate on erythromycin fermentation}

Figure 2A showed that the concentration of soluble phosphate in the sterilized medium was increased to approximately $0.1 \mathrm{~g} / \mathrm{L}$ after potassium dihydrogen phosphate was added. It was observed that the concentrations of amino nitrogen and total sugar under strategy 1 fell to 0.16 and $13 \mathrm{~g} / \mathrm{L}$ at about $30 \mathrm{~h}$, respectively, which was about $18 \mathrm{~h}$ earlier than that of the control experiment (Figure 2B). It was reasonable to think that inorganic
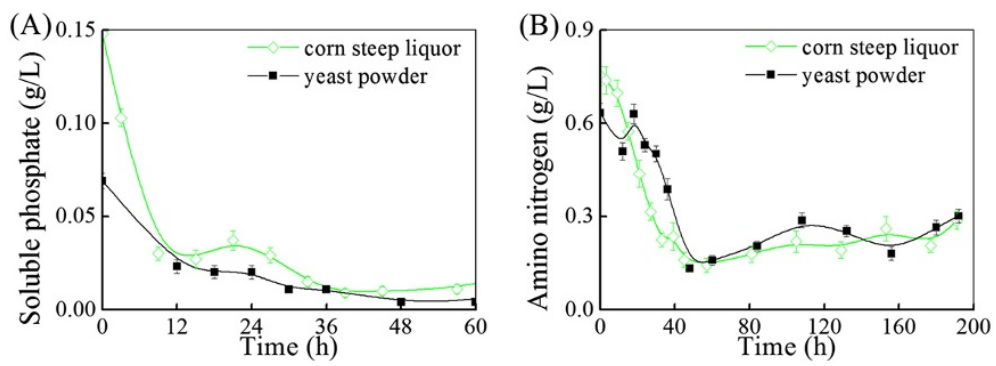

Figure 1 Comparison between the fermentation with yeast powder medium and that with corn steep liquor medium. (A) Soluble phosphate. (B) Amino nitrogen. 


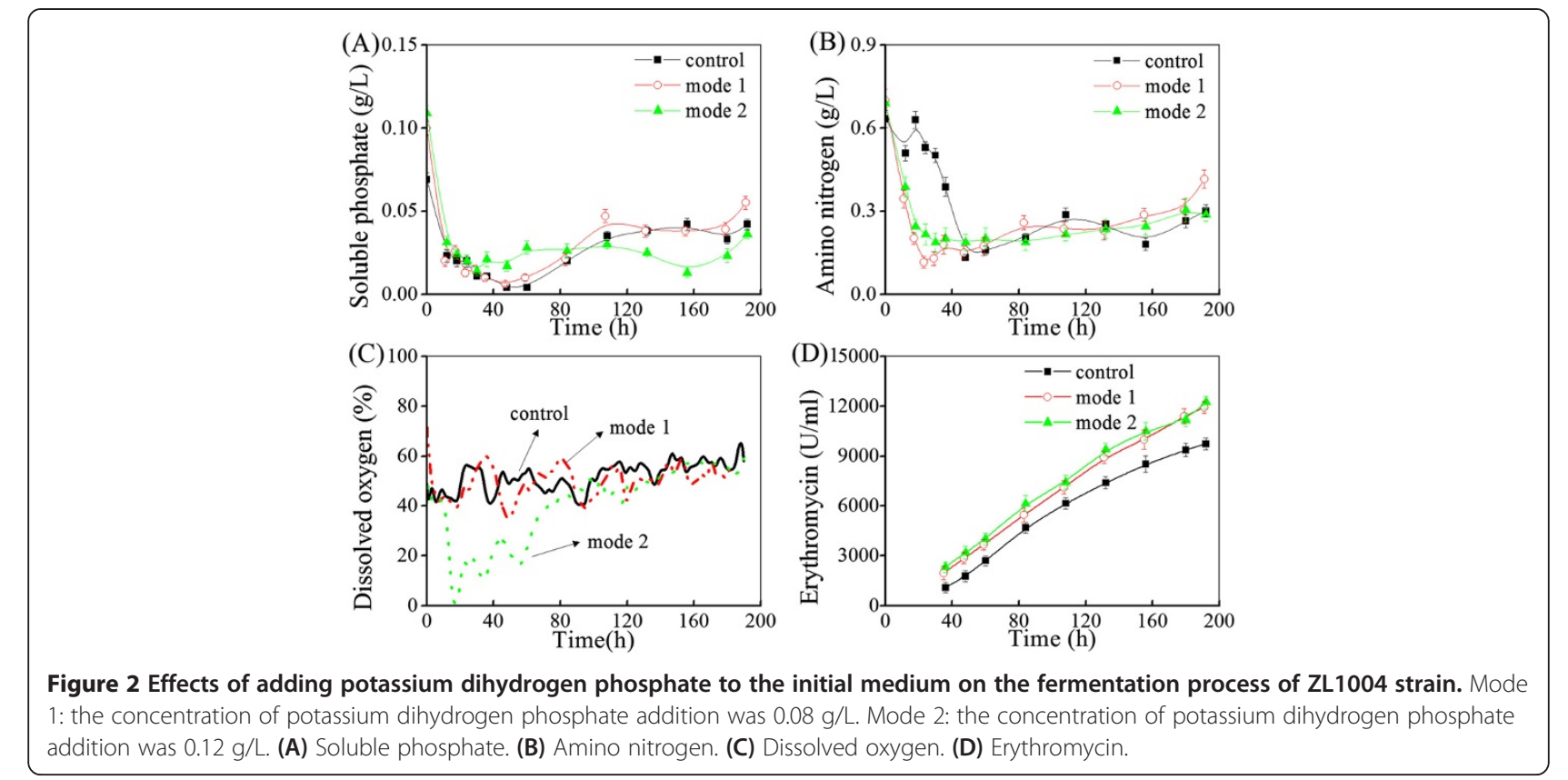

phosphate accelerated the consumption of amino nitrogen and starch. The maximal PMVs of mode $1(37 \%)$ and mode $2(42 \%)$ were slightly higher than that of the control (34\%). It was also observed that the dissolved oxygen concentration (DO) of mode 2 was dramatically low (approximately $10 \%$ ) during 15 to $20 \mathrm{~h}$ which coincided with the relatively high phosphate feed (Figure 2C). All these data supported that the metabolic activity of the cell was improved by the addition of potassium dihydrogen phosphate. Figure 2D showed that the erythromycin production under mode 1 and mode 2 were 11,918 and $12,230 \mathrm{U} / \mathrm{mL}$, which were increased by $22.3 \%$ and $25.5 \%$, respectively, as compared with the control. It should be noted that the biosynthesis rate of erythromycin before $36 \mathrm{~h}$ was significantly improved by the addition of inorganic phosphate. It was reported that the low level of free amino acids would expand the ppGpp pool in the starving cells and the ppGpp concentration was closely linked with the onset of antibiotic biosynthesis [21-23]. The possible explanation for the increase of erythromycin titer was that the high consumption rate of amino nitrogen after phosphate feed resulted in the starvation of amino nitrogen and ultimately triggered erythromycin biosynthesis earlier. Moreover, the key intermediates of primary metabolism should be increased since phosphate enhanced the primary metabolism and these intermediates provided more precursors of erythromycin [8]. We also found that the broth viscosity became too viscous under mode 2 , suggesting that phosphate was overfed (In mode 2, the final concentration of potassium dihydrogen phosphate in the initial medium was as high as $0.12 \mathrm{~g} / \mathrm{L}$ ).

\section{The effects of feeding potassium dihydrogen phosphate on the fermentation process}

Figure 3A showed that the concentration of soluble phosphate under strategy 2 was similar with that of the control
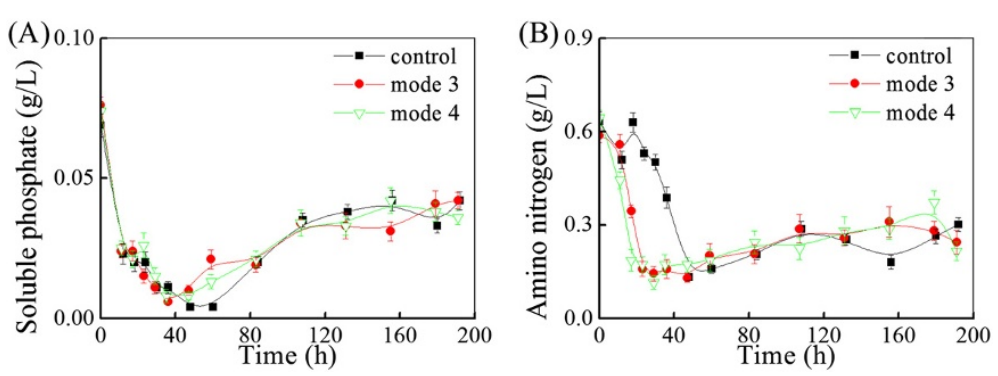

Figure 3 Effects of feeding potassium dihydrogen phosphate on the fermentation process of ZL1004 strain. Mode 3: the solution of potassium dihydrogen phosphate $(10 \mathrm{~g} / \mathrm{L})$ was fed at the rate of $10 \mathrm{~g} / \mathrm{h}$. Mode 4: the solution of potassium dihydrogen phosphate (10 g/L) was fed at the rate of $20 \mathrm{~g} / \mathrm{h}$. (A) Soluble phosphate. (B) Amino nitrogen. 
during 6 to $24 \mathrm{~h}$. Figure 3B showed that the concentration of amino nitrogen of mode 3 and mode 4 reached the lowest level at 24 and $18 \mathrm{~h}$, respectively, which were earlier than that of the control $(48 \mathrm{~h})$. These data further confirmed that phosphate accelerated the consumption of amino nitrogen. This phenomenon reflected that phosphate was the limiting nutrient for the cell growth and feeding potassium dihydrogen phosphate in the early phase of fermentation could alleviate the phosphate limitation.

The erythromycin production under mode $3(12,077$ $\mathrm{U} / \mathrm{mL})$ and mode $4(11,734 \mathrm{U} / \mathrm{mL})$ were increased by $24.0 \%$ and $20.4 \%$, respectively, as compared with that of the control. These data showed that feeding inorganic phosphate to improve the consumption rate of amino nitrogen was an effective way to improve erythromycin production. In our further study, feeding strategy was tested on 500-L fermenter scale.

\section{Feeding potassium dihydrogen phosphate on 500-L fermenter scale}

As shown in Figure 4, the consumption rate of amino nitrogen in 500-L fermenter was enhanced by feeding potassium dihydrogen phosphate. The PMV was significantly improved before $36 \mathrm{~h}$. These results were well consistent with the data acquired in 50-L fermenter. The erythromycin production with feeding potassium dihydrogen phosphate was reached to $11,839 \mathrm{U} / \mathrm{mL}$, which was $17.3 \%$ higher than that of control $(10,089 \mathrm{U} / \mathrm{mL}$, without feeding potassium dihydrogen phosphate). Hence, feeding potassium dihydrogen phosphate with amino nitrogen consumption as indicator was successfully scaled up from $50-\mathrm{L}$ fermenter to $500-\mathrm{L}$ fermenter.

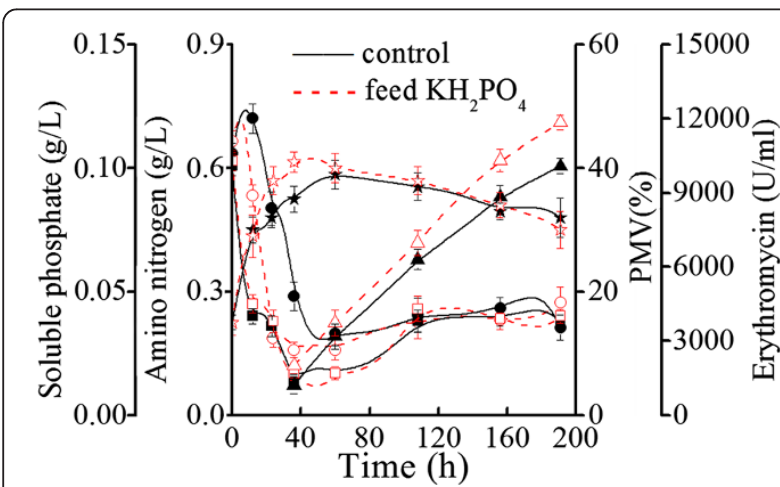

Figure 4 Effects of feeding potassium dihydrogen phosphate on the fermentation in $\mathbf{5 0 0 -} \mathrm{L}$ fermenter. Solid symbol indicates control; open symbol indicates feed $\mathrm{KH}_{2} \mathrm{PO}_{4}$. Black and red squares indicate soluble phosphate; black and red circles indicate amino nitrogen; black and red stars indicate PMV; and black and red triangles indicates erythromycin.
The erythromycin fermentation with condensed soy protein as easily metabolized nitrogen source

With the aim to further decrease the soluble phosphate level during the late phase of fermentation, condensed soy protein was selected as the nitrogen source since its phosphorus content $(0.9 \%)$ was much lower as compared with that of yeast powder (1.2\%) and corn steep liquor (1.5\%). The protein content of condensed soy protein was $65.0 \%$. Figure 5 showed that the consumption rate of amino nitrogen with feeding potassium dihydrogen phosphate was higher than that of the control. The PMV was also improved by feeding potassium dihydrogen phosphate. The erythromycin production was reached to $11,231 \mathrm{U} / \mathrm{mL}$, which was increased by $13.0 \%$ as compared with the control $(9,941 \mathrm{U} / \mathrm{mL})$. The material cost for erythromycin production in the medium with condensed soy protein $(3.59 \times$ $10^{-8}$ yuan/U) was cheaper than that in the medium with corn steep liquor $\left(3.94 \times 10^{-8}\right.$ yuan/U) and yeast powder $\left(3.78 \times 10^{-8}\right.$ yuan/U). Considering the relatively low price of condensed soy protein and the relatively high erythromycin titer, the combination of feeding potassium dihydrogen phosphate with condensed soy protein as nitrogen source showed a better prospective use on the industrial scale.

\section{Conclusions}

In this paper, a stable nitrogen source, yeast powder was introduced into erythromycin industrial fermentation medium in the place of the unstable nitrogen source, corn steep liquor. Considering the relatively low phosphorus content of yeast powder, a strategy of adding and feeding potassium dihydrogen phosphate was tested and the results showed that phosphate could enhance the cell growth in the early phase of fermentation and ultimately improved the erythromycin production. This strategy of feeding

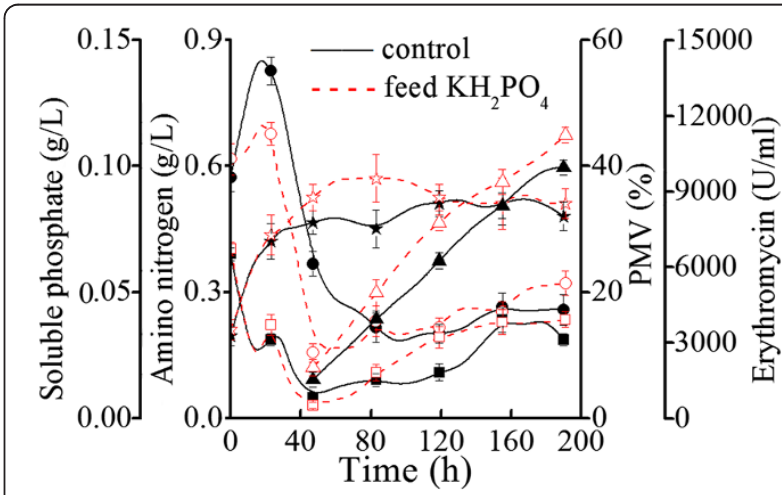

Figure 5 Effects of potassium dihydrogen phosphate on the fermentation process of condensed soy protein. Solid symbol, control; open symbol, feed $\mathrm{KH}_{2} \mathrm{PO}_{4}$. Black and red squares indicate soluble phosphate; black and red circles indicate amino nitrogen; black and red stars indicate PMV; and black and red triangles indicates erythromycin. 
phosphate was successfully applied to 500-L fermenter. The further fermentation with a low-phosphorus-content nitrogen source (condensed soy protein) supported that phosphate feed combined with rational nitrogen regulation was an effective way to improve erythromycin production.

\section{Competing interests}

The authors declare that they have no competing interests.

\section{Authors' contributions}

QZ, YC, and MH were in charge of the experiments and paper writing. YG participated in the experiments in the 500-L fermenter. JC and YPZ directed the study as the tutors. All authors read and approved the final manuscript.

\section{Acknowledgements}

This work was financially supported by a grant from the Major State Basic Research Development Program of China (973 Program, No.2012CB721006), National Natural Science Foundation of China (No.21276081), National Scientific and Technological Major Special Project (Significant Creation of New drugs, No.2011ZX09203-001-03), and Research Fund for the Doctoral Program of Higher Education of China (No.20110074110015).

\section{Author details}

'State Key Laboratory of Bioreactor Engineering, East China University of Science and Technology, 130 Meilong Road, P.O. box 329, Shanghai 200237 , People's Republic of China. ${ }^{2}$ Food Science and Engineering College, Qingdao Agriculture University, Qingdao 266109, Shandong, People's Republic of China. ${ }^{3}$ Yichang HEC Biochem. Co. Ltd, Yichang 443300, Hubei, People's Republic of China.

Received: 31 March 2014 Accepted: 14 August 2014

Published online: 12 September 2014

\section{References}

1. Mironov VA, Sergienko OV, Nastasyak IN, Danilenko VN (2004) Biogenesis and regulation of biosynthesis of erythromycins in Saccharopolysplra erythraea. Appl Biochem Microbiol 40:613-624

2. Castaldo RS, Celli BR, Gomez F, LaVallee N, Souhrada J, Hanrahan JP (2003) A comparison of 5-day courses of dirithromycin and azithromycin in the treatment of acute exacerbations of chronic obstructive pulmonary disease. Clin Ther 25:542-557

3. Weber JM, Cernota WH, Gonzalez MC, Leach BI, Reeves AR, Wesley RK (2012) An erythromycin process improvement using the diethyl methylmalonate-responsive (Dmr) phenotype of the Saccharopolyspora erythraea mutB strain. Appl Microbiol Biotechnol 93:1575-1583

4. Wu JQ, Zhang QL, Deng W, Qian JC, Zhang SL, Liu W (2011) Toward improvement of erythromycin A production in an industrial Saccharopolyspora erythraea strain via facilitation of genetic manipulation with an artificial attB site for specific recombination. Appl Environ Microbiol 77:7508-7516

5. Zou X, Hang HF, Chu J, Zhuang YP, Zhang SL (2009) Enhancement of erythromycin A production with feeding available nitrogen sources in erythromycin biosynthesis phase. Bioresour Technol 100:3358-3365

6. El-Enshasy HA, Mohamed NA, Farid MA, El-Diwany Al (2008) Improvement of erythromycin production by Saccharopolyspora erythraea in molasses based medium through cultivation medium optimization. Bioresour Technol 99:4263-4268

7. Zou X, Hang HF, Chu J, Zhuang YP, Zhang SL (2009) Oxygen uptake rate optimization with nitrogen regulation for erythromycin production and scale-up from $50 \mathrm{~L}$ to $372 \mathrm{~m}^{3}$ scale. Bioresour Technol 100:1406-1412

8. Gao Y, Yuan YJ (2011) Comprehensive quality evaluation of corn steep liquor in 2-keto-L-gulonic acid fermentation. J Agric Food Chem 59:9845-9853

9. Chu J, Li YR (2006) Modern concepts of industrial fermentation. Chemical Industry Press, Bei Jing

10. Zhang SL, Chu J, Zhuang YP (2004) A multi-scale study of industrial fermentation processes and their optimization. Adv Biochem Eng Biotechnol 87:97-150
11. Reeve LM, Baumberg S (1998) Physiological controls of erythromycin production by Saccharopolyspora erythraea are exerted at least in part at the level of transcription. Biotechnol Lett 20:585-589

12. Chen JF, Shao JW, Zhang YX, Chen H, Guo YH (2007) Control of phosphate concentration on aminoglycoside antibiotic JI-20A fermentation. Microbiology 34:852-855

13. Li XB, Zhao GR, Yuan YJ (2005) A strategy of phosphorus feeding for repeated fed-batch fermentation of penicillin G. Biochem Eng 27:53-58

14. Chen Y, Wang ZJ, Chu J, Zhuang YP, Zhang SL, Yu XG (2013) Significant decrease of broth viscosity and glucose consumption in erythromycin fermentation by dynamic regulation of ammonium sulfate and phosphate. Bioresour Technol 134:173-179

15. Chen Y, Deng W, Wu JQ, Qian JC, Chu J, Zhuang YP, Zhang SL, Liu W (2008) Genetic modulation of the overexpression of tailoring genes eryK and ery $G$ leading to the improvement of erythromycin A purity and production in Saccharopolyspora erythraea fermentation. Appl Environ Microbiol 74:1820-1828

16. Zhang SL, Ye BC, Chu J, Zhuang YP, Guo MJ (2006) From multi-scale methodology to systems biology: to integrate strain improvement and fermentation optimization. Chem Tech Biotechnol 81:734-745

17. Beljkas B, Matic J, Milovanovic I, Jovanov P, Misan A, Saric L (2010) Rapid method for determination of protein content in cereals and oilseeds: validation, measurement uncertainty and comparison with the Kjeldahl method. Accred Qual Assur 15:555-561

18. Chen Y, Huang MZ, Wang ZJ, Chu J, Zhuang YP, Zhang SL (2013) Controlling the feed rate of glucose and propanol for the enhancement of erythromycin production and exploration of propanol metabolism fate by quantitative metabolic flux analysis. Bioprocess Biosyst Eng 36:1445-1453

19. Yuan AQ, Tao PF, Zhao FY (2004) Detection of phosphate in the solution of zincphosphate with molybdenum blue method. Anal Test Technol Instrum 10:251-253

20. Zou X, Hang HF, Chen CF, Chu J, Zhuang YP, Zhang SL (2008) Application of oxygen uptake rate and response surface methodology for erythromycin production by Saccharopolyspora erythraea. Ind Microbiol Biotechnol 35:1637-1642

21. Friga GM, Borbely G, Farkas GL (1981) Accumulation of guanosine tetraphosphate (ppGpp) under nitrogen starvation in Anacystis nidulans, a cyanobacterium. Arch Microbiol 129:341-343

22. Akinyanju J, Smith RJ (1979) Accumulation of ppGpp and pppGpp during nitrogen deprivation of the cyanophyte Anabaena cylindrica. FEBS Lett 107:173-176

23. Ochi K (1987) Metabolic initiation of differentiation and secondary metabolism by Streptomyces griseus: significance of the stringent response (ppGpp) and GTP content in relation to A factor. J Bacteriol 169:3608-3616

doi:10.1186/s40643-014-0015-7

Cite this article as: Zhang et al: The dynamic regulation of nitrogen and phosphorus in the early phase of fermentation improves the erythromycin production by recombinant Saccharopolyspora erythraea strain. Bioresources and Bioprocessing 2014 1:15.

\section{Submit your manuscript to a SpringerOpen ${ }^{\circ}$ journal and benefit from:}

- Convenient online submission

- Rigorous peer review

- Immediate publication on acceptance

- Open access: articles freely available online

- High visibility within the field

- Retaining the copyright to your article

Submit your next manuscript at $>$ springeropen.com 